
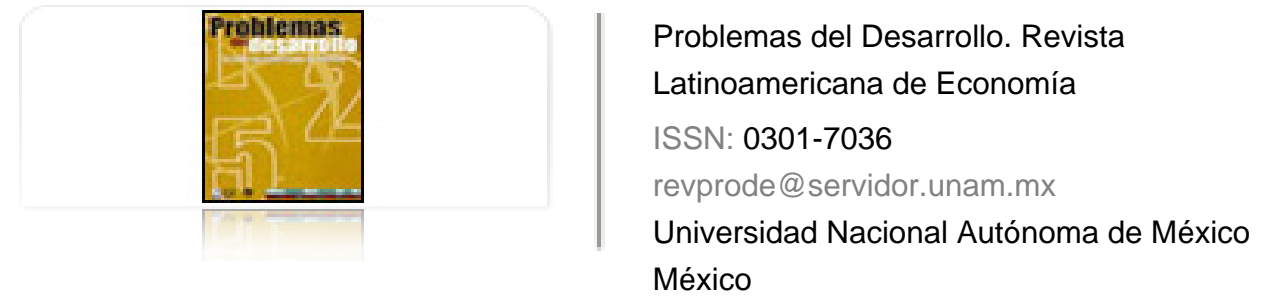

Depetris Guiguet, Edith; García Arancibia, Rodrigo; Rossini, Gustavo DESEMPEÑO COMPETITIVO DE ARGENTINA Y URUGUAY EN LA LECHE EN POLVO Problemas del Desarrollo. Revista Latinoamericana de Economía, vol. 40, núm. 157, abril-junio, 2009, pp. $163-187$

Universidad Nacional Autónoma de México

Distrito Federal, México

Disponible en: http://www.redalyc.org/articulo.oa?id=11820087003

Cómo citar el artículo

- Número completo

- Más información del artículo

Página de la revista en redalyc.org

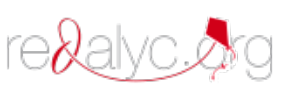

Sistema de Información Científica

Red de Revistas Científicas de América Latina, el Caribe, España y Portugal Proyecto académico sin fines de lucro, desarrollado bajo la iniciativa de acceso abierto 


\title{
DESEMPEÑO COMPETITIVO DE ARGENTINA Y URUGUAY EN La LECHE EN POLVO
}

\author{
Edith Depetris Guiguet* \\ Rodrigo García Arancibia** \\ Gustavo Rossini***
}

Fecha de recepción: 15 de enero de 2009. Fecha de aceptación: 4 de mayo de 2009.

\section{Resumen}

Los sectores lácteos de Argentina y Uruguay hacen importantes contribuciones tanto al producto interno bruto como a la generación de divisas provenientes de sus exportaciones. Al constituirse el MERCOSUR ambos países tenían excedentes exportables de leche en polvo entera y descremada, y aunque con similares condiciones del contexto externo impuestas por la integración y la evolución del mercado internacional, el comportamiento exportador no ha sido igual en las dos naciones. Esto lleva a cuestionar si ambos o alguno de ellos ha perdido competitividad en el comercio a partir del inicio de la integración, respuesta que se espera encontrar como resultado de esta investigación. Por tanto, este estudio tiene como objetivo conocer la evolución competitiva del comercio internacional de leche en polvo de Argentina y Uruguay en ese periodo. Para ello se utilizarán varios indicadores alternativos, probándose su consistencia a los efectos de verificar la coherencia de los resultados.

Palabras clave: competitividad, indicadores, leche en polvo entera, leche en polvo descremada y prueba de consistencia.

* Docente titular e investigadora, categoría I, del Departamento de Economía de la Facultad de Ciencias Económicas de la Universidad Nacional del Litoral, Argentina. Correo electrónico: eguiguet@fce.unl.edu.ar.

** Docente de Organización Industrial del Departamento de Economía de la Facultad de Ciencias Económicas de la Universidad Nacional del Litoral. Correo electrónico: rgarcia@fce.unl.edu.ar.

*** Docente de Econometría e investigador del Departamento de Economía de la Facultad de Ciencias Económicas de la Universidad Nacional del Litoral. Correo electrónico: grossini@fce.unl. edu.ar.

Este trabajo es resultado del proyecto PICTO núm. 36284 de la Agencia Nacional de Promoción Científica y Tecnológica-UNL, financiado por el BID 1728/OC-AR y CAID (UNL). 


\section{Abstract}

The dairy sectors in Argentina and Uruguay make important contributions both to the gross domestic product and to the generation of foreign exchange earned by their exports. In establishing the MERCOSUR both countries had exportable surpluses of powdered full-cream and skimmed milk, and although they have similar conditions of external context imposed by the integration and evolution of the international market, the export performance has not been the same for each of these countries. This raises the question of whether one or both of them have lost its competitiveness in trade since the beginning of integration, a response to which we expect to find as a result of this investigation. Therefore, this study aims at understanding the competitive evolution of the international trade in powdered milk in Argentina and Uruguay during that period. To do this several alternative indicators will be used, proving their consistency with the effects of verifying the coherence of the results.

Key words: competitiveness, indicators, powdered full-cream milk, powdered skimmed milk, consistency test.

\section{Résumé}

En Argentine et en Uruguay, la production laitière contribue dans une large mesure tant au produit interne brut comme à la génération de devises à travers l'exportation. Quand s'est constitué le MERCOSUR, les deux pays avaient des excédents exportables de lait en poudre et écrémé, et bien que placées dans des conditions similaires par le fusionnement et l'évolution du marché international, leurs exportations n'ont pas eu le même comportement. Cet état de fait conduit à se demander si les deux nations ou l'une d'entre elles est devenue moins compétitive commercialement à partir du début du fusionnement, question à laquelle cette recherche tente de répondre. C'est pourquoi cette étude prend pour objet l'évolution de la compétitivité commerciale des laits en poudre argentins et urugayens sur le plan international durant cette période. Plusieurs indicateurs alternatifs seront employés, dont on mettra à l'épreuve la significativité de manière à vérifier la cohérence des conclusions.

Mots clés: compétitivité, indicateurs, lait entier en poudre entier, lait écrémé en poudre et preuve de significativité

\section{Resumo}

Os setores lácteos da Argentina e do Uruguai fazem importantes contribuições tanto ao produto interno bruto como à geração de divisas provenientes de suas exportações. Quando se constituiu o MERCOSUR os dois países tinham excedentes exportáveis de leite em pó integral e desnatado, e apesar de contar com similares condições do contexto externo impostas pela integração e a evolução do mercado internacional, o comportamento exportador tem sido igual nas duas nações. Isto leva a questionar se ambos ou algum deles terá perdido competitividade no comércio a partir do início da integração, resposta que se espera encontrar como resultado desta investigação. Por tanto, este estudo tem como objetivo conhecer a evolução competitiva do comércio internacional de leite em pó da Argentina e do Uruguai nesse período. Para isso utilizar-se-ão vários indicadores alternativos, provando-se sua consistência com o fim de verificar a coerência dos resultados.

Palavras-chave: competitividade, indicadores, leite em pó integral, leite em pó desnatado e prova de consistência.

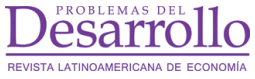

Vol. 40, núm. 157, abril-junio / 2009 


\section{Introducción}

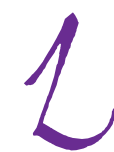

os sectores lácteos de Argentina y Uruguay tienen importancia para su desarrollo tanto por el valor agregado interno que generan como por las divisas que ingresan por las exportaciones. Para el primero, la producción lechera primaria representa cerca de $8 \%$ del producto bruto agropecuario y la industrial casi $2 \%$ del producto bruto manufacturero. Ha llegado a canalizar en sus exportaciones hasta $20 \%$ de la producción nacional.

Para Uruguay, la lechería tiene más relevancia, pues engloba casi 15\% de su producto bruto agropecuario, llegando a exportar hasta $60 \%$ de la producción. En ambos casos los principales productos exportables han sido leche en polvo, mantequilla y quesos.

En 1991, después de la firma del Tratado de Asunción, que dio nacimiento al MERCOSUR, ${ }^{1}$ ambos países eran los únicos del grupo con excedentes lácteos exportables de relevancia, pero el comercio se venía realizando mayormente sobre la base de acuerdos bilaterales con concesiones y preferencias. Como Brasil y Paraguay actuaban como netos importadores, la integración abría un mercado ampliado relevante para los exportadores. A partir de 1995 Argentina y Brasil liberaron sus fronteras para el comercio intrabloque de lácteos, mientras que Uruguay y Paraguay establecieron una lista de productos para los cuales se haría una desgravación lineal hasta llegar a cero en cinco años. Al mismo tiempo se adoptó un arancel externo común con el criterio de tratar de corregir distorsiones y permitir desarrollar condiciones de competitividad mínima para el sector lácteo en los países signatarios.

A pesar de este contexto común, los dos exportadores mostraron comportamientos diferentes en su comercio exterior de lácteos, lo que se pone en evidencia en el caso de la leche en polvo. Mientras que Argentina se ha especializado en leche en polvo entera (LPE), Uruguay había sido tradicionalmente más fuerte en leche en polvo descremada (LPD), con cambios significativos en los últimos años.

Las exportaciones argentinas de LPE alcanzaban al comienzo de los años noventa 24 millones de dólares y las de Uruguay 4.6 millones. Cuando efectivamente entra en funciones el MERCOSUR en 1995 las mismas habían crecido a casi 136 millones y 10.4 millones respectivamente. Pasada una década, Argentina exporta unos 367 millones mientras que Uruguay lo hace por 34 millones. Ambos países muestran un pronunciado incremento después de 2003. La evolución para el periodo de integración en el MERCOSUR de las dos naciones, por tipos de productos y destinos se muestra en las gráficas 1 y 2 .

1 El tratado se firma en 1991 y la aplicación efectiva se da desde 1995. Para más detalle del proceso con respecto a los lácteos, véase Depetris de Guiguet, E. y Cappellini, O. (1998). 
Gráfica 1

Exportaciones de LPE argentinas y uruguayas totales y al MERCOSUR
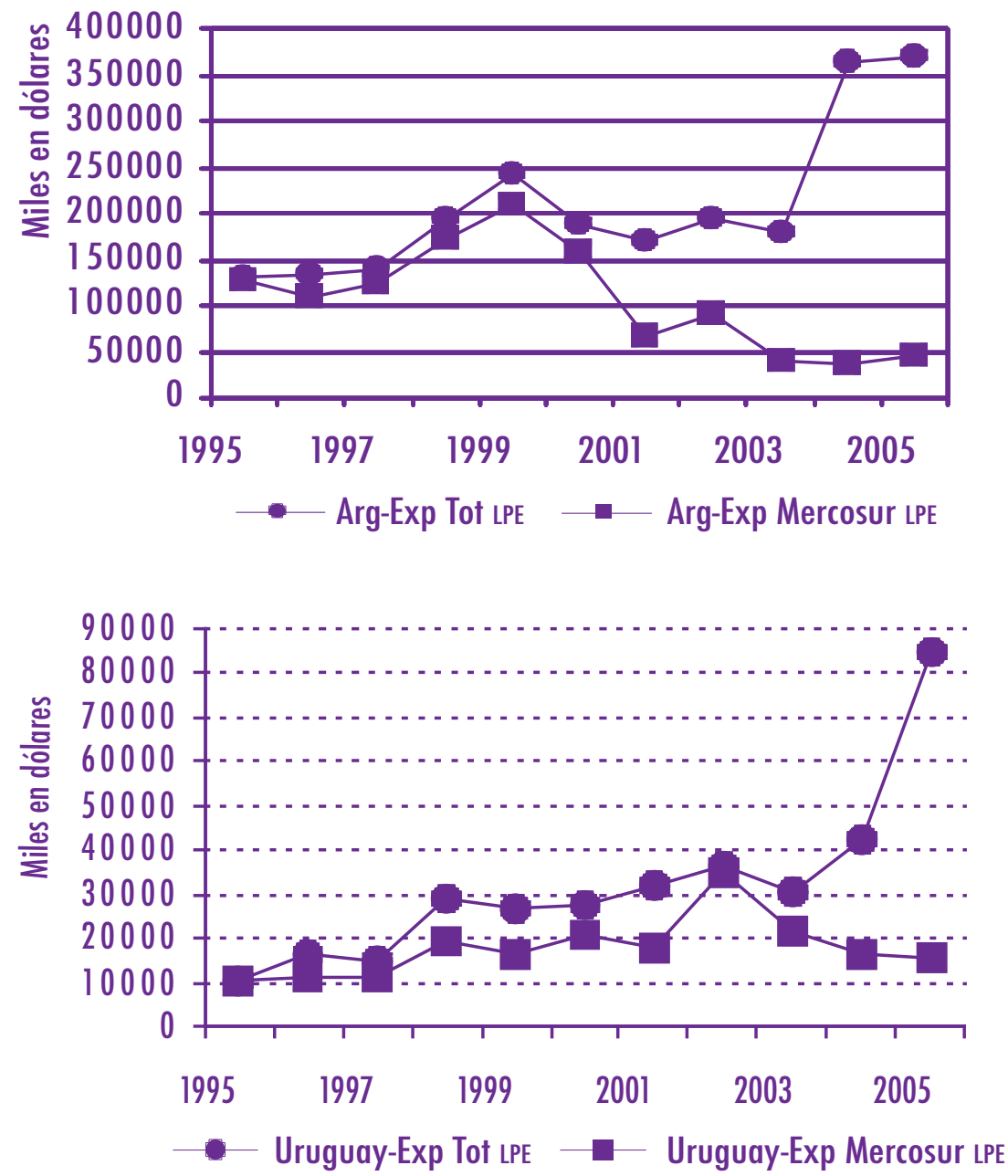

Fuente: Elaboración propia según datos del INDEC (Argentina) y del Sistema Lucía (Uruguay).

En LPD Argentina muestra una tendencia decreciente en todo el periodo, ya que en 1990 exportaba por un total de 39 millones, en 1995 las había reducido a 38 millones de dólares, y a 33 millones una década después. Uruguay, en cambio, llega a incrementarlas hasta los 26 millones y luego bajan hasta 2004, momento en que se revierte la tendencia. 
Gráfica 2

Exportaciones de LPD uruguayas totales y al MERCOSUR
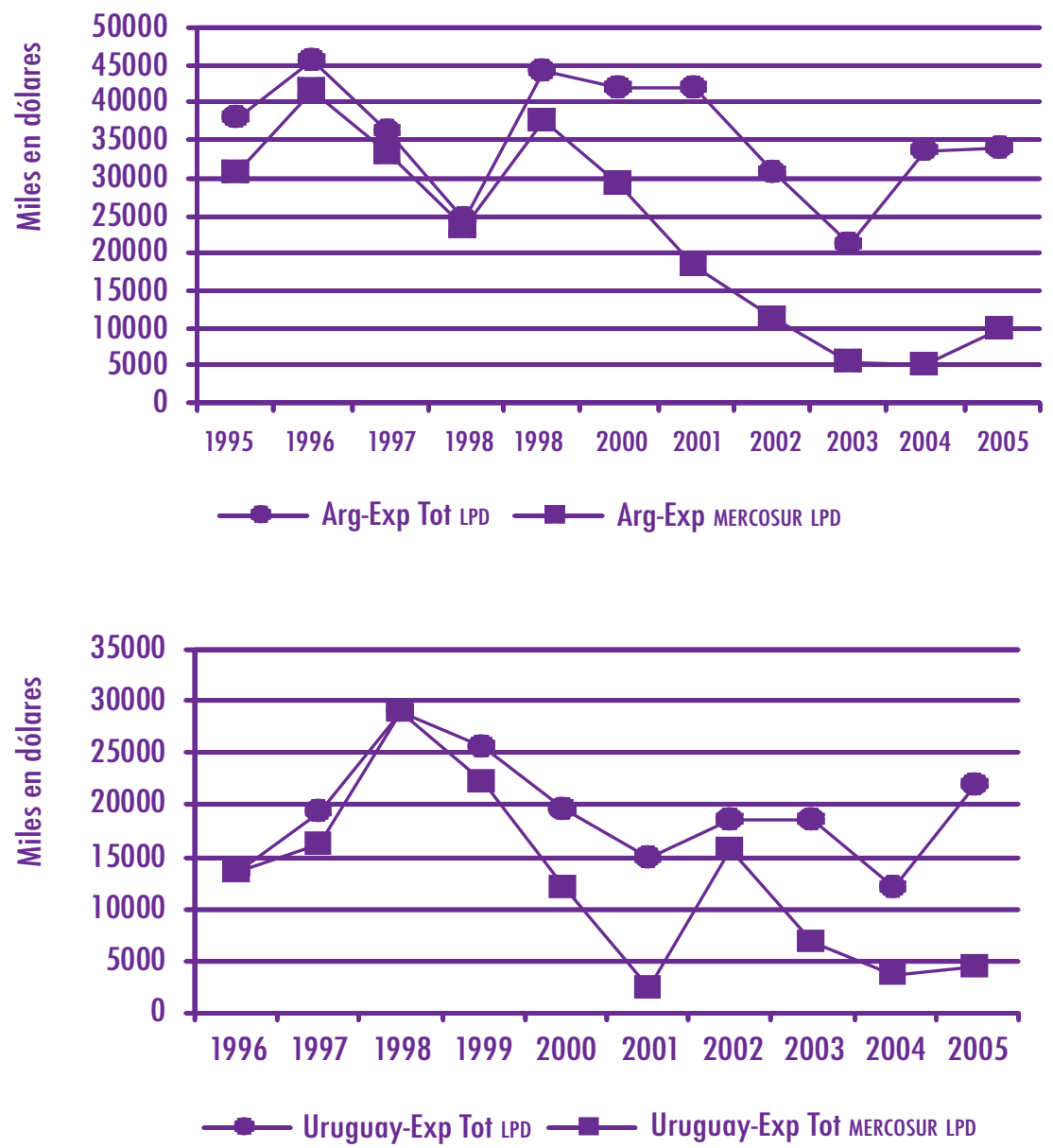

Fuente: Elaboración propia con datos del INDEC (Argentina) y del Sistema Lucía (Uruguay).

Como se observa en las gráficas precedentes, desde 1995 hasta 1999 la mayor parte de las exportaciones de los dos tipos de leches en polvo tuvieron como destino el MERCOSUR. En el caso de Argentina representaron para ambas más de $80 \%$ de los valores exportados y en Uruguay de 80\% en LPD y más de 60\% en LPE. Pero a partir de la devaluación brasileña, su demanda se redujo y los socios exportadores debieron buscar mercados alternos fuera del bloque. Se exponen entonces de lleno a la competencia internacional en la busca de nuevos mercados o incursionan en algunos con los que se tenían previamente acuerdos comerciales. 
Así es que no obstante las condiciones similares del contexto externo impuestas por la integración en el mismo bloque y evolución del mercado internacional, el comportamiento exportador en cada uno ha tenido muchas fluctuaciones y no ha sido igual para cada país. Esto lleva a cuestionar si ambos o alguno de ellos han perdido competitividad en el comercio de estos productos a partir del inicio de la integración, respuesta que se espera encontrar como resultado de esta investigación.

Por tanto, este estudio tiene como objetivo conocer la evolución competitiva del comercio internacional de leche en polvo de Argentina y Uruguay entre 1990 y 2005. Para ello se utilizarán varios indicadores alternativos, probándose su consistencia a los efectos de verificar la coherencia de los resultados.

\section{Ventajas competitivas reveladas: metodología para su medición}

En los estudios de competitividad se han propuesto diferentes herramientas metodológicas para describir su evolución, así como los patrones revelados en un periodo en particular. Si resulta poco consensuada la definición del término "competitividad", mayor complicación trae aparejado el problema de su medición. Por lo común se elige el concepto de acuerdo con la conveniencia de lo que se pretende medir, y de las variables que entran en el cómputo del indicador que se proponga, para así tener una medida cuantitativa representativa. En los estudios de competitividad internacional una de las medidas más utilizadas es el denominado indicador de ventajas comparativas reveladas (VCR) de Balassa (1965). Aunque en su origen tuvo una acepción más restringida, después se ha utilizado como una medida de ventajas o desventajas competitivas, indicando con ello la aptitud de un país de competir en el ámbito internacional en un producto en particular. La mayor amplitud conceptual se justifica por la construcción misma del indicador, pues sus términos reflejan los efectos de las restricciones existentes en el comercio u otras formas de intervención gubernamental (Scott y Vollrath, 1992; Pitts y Lagnevik, 1997; Ferto y Hubbard, 2001).

Adicional al índice de Balassa, otros autores (Vollrath, 1991; Laursen, 1998; Ferto y Hubbard, 2001) han propuesto otras medidas para evaluar la competitividad, algunas para tratar de corregir las limitaciones del primero y otras con aportes de nuevos elementos, incluyendo en algunos casos medidas convencionalmente utilizadas para estudios de especialización. Si bien todos los indicadores intentan medir el mismo concepto, su construcción difiere, aun cuando suelen utilizarse datos similares para su cómputo.

La cuestión es si ellos indistintamente conducen a similares resultados, demarcando de forma inequívoca y consistente la posición ventajosa o desventajosa de un país. Con este fin se aplican pruebas de consistencia en tres sentidos: en términos del 
resultado binario ventajas-desventajas (sentido dicotómico), como medidas de grado del desempeño competitivo de acuerdo con el valor especifico que asumen (sentido cardinal) y en función del ordenamiento establecido por cada uno de ellos, considerando el rango entre los años en que se revelan las mayores ventajas hacia aquellos en los que se muestran desventajas competitivas (sentido ordinal).

\section{Índice de Balassa (IB)}

El primer indicador que se considerará es el denominado índice de Balassa (IB) de ventajas comparativas reveladas (1965). Esta propuesta se asocia a la imposibilidad metodológica de obtener una medida exacta de las ventajas comparativas de un país que, de acuerdo con la concepción ricardiana, requeriría tener conocimiento de las circunstancias pre-comercio de la economía en autarquía. A pesar de ello, Balassa construye una medida aproximada (IB) con el supuesto de que las ventajas comparativas de un determinado sector son "reveladas" por los datos de los flujos comerciales (i.e.post comercio). Esta construcción se mantiene sin descuido en el espíritu de la obra de Ricardo y de la teoría de comercio internacional del modelo Herscher-Ohlin basados en modelos de dos bienes y dos países. Dado que los flujos comerciales post comercio sobre los que se basa el cómputo resultan de un conjunto de factores adicionales a la dotación factorial propia de un país, como son los efectos de medidas gubernamentales y otros, el IB también puede interpretarse como medida de ventaja competitiva.

Como caso particular, el indicador puede expresarse como una relación entre la participación mundial del país $i$ en las exportaciones del producto $l$ y la participación mundial del país en el total de productos exportados, específicamente:

$$
I B_{i / j, l}=\frac{\left(x_{i, l} / X_{i}\right)}{\left(x_{j, l} / X_{j}\right)} \equiv \frac{\left(x_{i, l} / x_{j, l}\right)}{\left(X_{i} / X_{j}\right)}
$$

donde $x_{i, l}$ denota el valor de las exportaciones del bien $l$ que realiza el país $i$, análogamente $x_{i, l}$ denota las exportaciones del bien $l$ realizadas por el conjunto de países $j$ de referencia y $X_{k}$ el valor de las exportaciones totales realizadas por $k$, para $k=i, j$.2

2 El índice de Balassa tradicional considera al conjunto de productos manufacturados como grupo de países de referencia; sin embargo, para el caso de lácteos, los índices son consistentes de acuerdo con estas dos referencias (Depetris et al., 2008a). Para un análisis de indicadores aplicado a lácteos en Argentina que se toman como al conjunto de productos manufacturados véase como referencias Depetris et al., 2008b. 
En este estudio $i$ corresponde a Argentina (A) o Uruguay (U), el conjunto de países de referencia $j$ será el total mundial (M) y $l$ denotará por un lado la leche en polvo entera ( $l=$ LPE) y, por otro, la leche en polvo descremada $(l=$ LPD). Por tanto, el IB sería la participación relativa de las exportaciones de leche en polvo medida en términos de la participación de las exportaciones mundiales.

De (1) se deduce que el índice asumirá valores desde 0 hasta $+\infty$, dado que tanto el numerador como el denominador pueden asumir valores desde 0 hasta $+\infty$. Específicamente, el límite superior del intervalo queda determinado por el inverso de la participación de las exportaciones totales del país en las exportaciones mundiales totales, i.e. $\mathrm{IB}_{i / j, l} \in\left[0, X_{j} / X_{i}\right]$ (De Benedictis, 2002:5). Por tanto, el límite superior tenderá a infinito si la participación en las exportaciones totales tiende a ser nula.

La interpretación más aceptada del valor que asume el IB es el sentido de demarcación entre ventajas/desventajas competitivas dado por el valor en el que la participación de tal producto en el país es idéntica a la participación que tiene en el conjunto de países de referencia, en este caso constituido por el total mundial: si es mayor a uno se interpreta la existencia de ventajas comparativas-competitivas en el sector, mientras que cuando los valores son menores a la unidad se está en presencia de desventajas comparativas-competitivas.

Si bien la expresión del IB presentada en (1) compara las exportaciones de un sector con el total de bienes exportados y referenciando al país en cuestión con el total mundial, las posibilidades de este indicador son más amplias. El cómputo del mismo permite tomar un sector o bien y compararlo con cualquier otro dentro del mismo país, como también tomar las exportaciones de un producto de un país de interés o bloque regional, y compararlo con sus similares de un conjunto de naciones de referencia. De este modo, resulta evidente que la construcción del IB se basa en el marco teórico de dos países/dos bienes del modelo neoclásico del comercio internacional.

Dado que el índice (1) puede variar por cambios en la participación mundial que tiene el país en las exportaciones de la leche en polvo (numerador) o bien por variaciones en la participación mundial de exportaciones totales (denominador), o ambos a la vez; comparar directamente los valores de RCA obtenidos para un país con los de otros puede llevar a conclusiones erróneas. Ello es porque la importancia relativa de un determinado sector en términos de la competitividad internacional dependerá de la estructura de exportaciones del total de productos que tiene cada país y, al diferenciarse ésta, el valor cardinal del indicador reflejará diversas cantidades sin que con ello se asigne una mayor competitividad en un país que en otro. Esto si se piensa en que ambos valores se encuentran del mismo lado respecto a la unidad, ya que, en 
caso contrario, la comparación de acuerdo con el criterio de demarcación ventajasdesventajas comparativas resultaría comprensible.

\section{Índice de ventaja relativa del comercio (IVRC)}

Ya que el IB sólo se basa en el valor de las exportaciones brutas, las consideraciones por el lado de la demanda son omitidas. Para su inclusión resulta necesario incorporar los flujos de las importaciones, pensando así la competitividad en términos de las exportaciones netas. Vollrath (1991) propone una versión neta del índice de ventajas comparativas reveladas, aplicada en ciertos trabajos como el de Eiteljörge et al. (1999) para estudios de competitividad dentro de la Unión Europea. Antes de definir este indicador "neto", es necesario presentar la versión de importaciones del Balassa (índice de ventaja revelada de importaciones), la que viene dada por

$$
\operatorname{VRI}_{A / j, l}=\frac{\left(m_{i, l} / M_{i}\right)}{\left(m_{j, l} / M_{W}\right)}
$$

En donde $m_{A, l}$ y $m_{W, l}$ son el análogo definido anteriormente para la expresión (1), pero para el caso de las importaciones, donde valores mayores que uno representan una mayor participación de las importaciones del producto, que en promedio tiene el conjunto de países de referencia y, por tanto, revelaría desventajas comparativas en tal sector.

Luego definimos el índice de ventaja relativa del comercio como la diferencia entre (1) y (2):

$$
\operatorname{IVRC}_{i / j, l}=\frac{\left(x_{i, l} / X_{i}\right)}{\left(x_{j, l} / X_{j}\right)}-\frac{\left(m_{A, l} / M_{A}\right)}{\left(m_{j, l} / M_{W}\right)}
$$

Los valores que puede asumir (3) quedan determinados por los límites superiores de IB y VRI, i.e. $\operatorname{IVRC}_{i / j, l} \in\left[-\frac{M_{j}}{M_{i}}, \frac{X_{j}}{X_{i}}\right]$ para $i=\mathrm{A}, \mathrm{U}$ y $j=$ Mundo. A diferencia del IB, que es siempre positivo, con el IVRC la ventaja competitiva se revela por medio del signo: si es positivo (negativo) se revelan ventajas (desventajas) competitivas para el país $i$ en el comercio mundial bien $l$.

\section{Índice de Michaely modificado (IMM)}

El índice de Michaely (1984) es una medida de exportaciones netas relativas en un sector determinado y es utilizado en la mayoría de los estudios de especialización 
sectorial del comercio internacional en general (Laursen, 1998) y de lácteos en particular (Depetris et al., 2008b). A su vez este indicador ha sido expresado en relación con las participaciones comerciales combinadas (Guel y Martin, 1995; Fertö I. y Hubbard, 2001). Una de las ventajas en utilizar esta variante del indicador es que se reduce el número de decimales relevantes, ya que al analizar la participación de un sector tan específico como el de lácteos en relación con el total de commodities del país, las participaciones resultan demasiado pequeñas, lo que es arrastrado por el valor del indicador. Al dividir por la participación comercial (participación de las exportaciones más participación de importaciones), el valor que asume el indicador es mayor, reduciendo la cantidad de ceros decimales previos al primer valor no nulo. A esta formulación la denominaremos índice de Michaely modificado, la que viene dada por

$$
\operatorname{IMM}_{i, l}=\left(p^{x}{ }_{i, l}-p^{m}{ }_{i, l}\right) /\left(p^{x}{ }_{i, l}+p^{m}{ }_{i, l}\right)
$$

donde $p_{i, l}^{x}$ y $p_{i, l}^{m}$ representan las participaciones del valor de las exportaciones e importaciones del bien $l$ respecto del valor total de las exportaciones e importaciones que realiza el país $i$, respectivamente. En otros términos:

$$
p_{i, l}^{x}=\frac{x_{i, l}}{X_{i}} ; p^{x}{ }_{i, l}=\frac{m_{i, l}}{M_{i}} \text { para } i=\mathrm{A}, \mathrm{U} \mathrm{y} l=\mathrm{LPE}, \mathrm{LPD}
$$

A diferencia de los otros índices, el IMM está acotado en el intervalo -1 y 1, donde un valor positivo indicaría que el país $i$ es competitivo en el bien o sector $l$, caso contrario si es negativo. Si fuera nulo se estaría en presencia de "perfecta similaridad" (Laursen, 1998).

\section{Índice de Lafay (IL)}

Como mejora del índice de Michaely, Lafay (1992) propone un indicador a fin de medir el desempeño comercial y la especialización en determinados sectores, siendo a su vez comparado con los indicadores alternativos de ventajas comparativas (Laursen, 1998). Puede demostrarse que este indicador permite un mayor control de las distorsiones introducidas por las fluctuaciones macroeconómicas, al considerar la diferencia entre balanza comercial sectorial normalizada y la balanza comercial global, ponderando por la importancia respectiva en el comercio (Zaghini, 2003; 9:11). 
Con la notación antes introducida, el índice de Lafay para el producto $l$ puede escribirse $\mathrm{como}^{3}$

$$
\mathrm{IL}_{i, l}=\left[\frac{x_{i, l}-m_{i, l}}{x_{i, l}+m_{i, l}}-\frac{X_{i}-M_{i}}{X_{i}+M_{i}}\right] \cdot \frac{x_{i, l}-m_{i, l}}{X_{i}+M_{i}} \cdot 100
$$

Como puede observarse en (5), aquí la ventaja competitiva del país $i$ en la producción y comercio del bien $l$ es medida como la desviación de la balanza comercial normalizada del sector $l$, de la balanza total (normalizada), ponderada por la participación del volumen comerciado del bien $l$ en el total.

En resumen, tenemos dos indicadores (1) y (3) de ventajas comparativas en el sentido de que su construcción es consistente con las dimensiones inherentes del de dos países/dos bienes (Vollrath, 1991.); y dos indicadores (4) y (5) específicos de estudios de especialización, en el sentido de que quedan expresados en términos de comparaciones de la balanza comercial de un sector respecto a la balanza comercial global. A fin de hacerlos comparativos, los dos primeros indicadores consideran al total de productos comerciados y al total mundial, como referencia de conjunto de bienes y conjunto de países, respectivamente. Pero dadas estas diferencias ies posible tomarlos en cuenta como indicadores alternativos del desempeño competitivo de un sector dentro de un país?

En términos teóricos cada indicador se puede interpretar como medida de competitividad dada la amplitud del término y el poco consenso sobre su significado específico, adoptando una cierta postura conformable con lo que se está midiendo. Pero con mayor generalidad, cada medida pretende representar el desempeño de una nación en el comercio del bien, ya sea afirmando que el sector se está especializando, o que tiene ventajas comparativas, o bien un desempeño competitivo favorable. Es decir, que al afirmar que un país tiene ventajas competitivas en un determinado sector, implícitamente hay una componente relacionada al costo relativo de los factores, dada la dotación de la nación; pero también un factor "artificial" que comprende los efectos causados por las distintas medidas políticas y comerciales. A su vez, si el país tiene tales ventajas resulta razonable suponer que se especializa en la producción del bien en cuestión para los fines del comercio.

3 Una construcción similar es el denominado índice de contribución a la balanza comercial. 


\section{Pruebas de consistencia}

Las pruebas de consistencias cardinal, ordinal y dicotómica fueron presentadas por Ballance et al. (1987) a fin de comparar el tradicional índice de Balassa con otros indicadores de desempeño en el comercio internacional. Recientemente se han aplicado a trabajos del sector agroalimentario (Fertö et al., 2002) y lácteo en particular (Depetris et al., 2008b) tomando diferentes "paquetes" de indicadores del comercio como medidas alternativas. En algunos se realizan sobre los distintos bienes o sectores para un año determinado, mientras que en otros se toma un determinado sector y la prueba se hace sobre los distintos años considerados. Este último enfoque es el que adoptamos en el presente trabajo.

La prueba de consistencia cardinal consiste en calcular el coeficiente de correlación entre los valores de los indicadores en todos los años del periodo considerado. La prueba de consistencia ordinal se realiza mediante el cómputo de los coeficientes de correlación por rangos de Spearman. Esta prueba permite revisar hasta qué punto los valores de los indicadores son consistentes cuando se realiza un ordenamiento entre años según revelen mayores ventajas competitivas o menores, o incluso en los que muestran desventajas competitivas.

Por último, consideramos la prueba de consistencia dicotómica como aquella que mide la proporción de casos (años) en los cuales cada par de indicador coincide respecto al posicionamiento competitivo del país en determinado sector, tomando el resultado binario ventajas versus desventajas.

\section{Análisis aplicado}

En el periodo analizado (1990-2005), Argentina y Uruguay han experimentado diferentes cambios en sus políticas comerciales y cambiarias. De acuerdo con la concepción adoptada en el presente estudio en relación con el término "competitividad", tales factores contribuyen a la reasignación de recursos entre los distintos sectores de un país, en el fortalecimiento competitivo por parte de algunos sectores productivos, y un debilitamiento relativo en otros. Es así que nos concentramos en la evolución de un producto lácteo en particular, la leche en polvo, tanto entera (LPE) como descremada (LPD). Por tanto, al estudiar un sector tan específico, no pretendemos abordar el cambio de los patrones de comercio inducido por variaciones en los diferentes factores que implican un mayor posicionamiento competitivo, sino estudiar la evolución de la competitividad medida por diferentes propuestas metodológicas. Además, desa-gregamos algunas componentes para determinar su peso relativo, asociadas al sector en particular dentro del país y a las relacionadas con la evolución de las 
exportaciones en general. Por último, se estudiará hasta qué puntos son consistentes los indicadores como medidas de demarcación, de ordenamiento año tras año o como medidas cardinales (i.e. de grado) considerando cada caso particular, es decir, Argentina-LPE, Argentina-LPD, Uruguay-LPE y Uruguay-LPD.

Los datos de exportaciones e importaciones de leche en polvo utilizados para obtener los indicadores de este trabajo se obtienen de la base de FAOSTAT para el periodo 1990-2005 y los valores de las exportaciones e importaciones totales de la base de UNCTAD.

\section{Resultados}

\section{Evolución de la competitividad en Argentina y Uruguay}

Como se vio en la Introducción, el comercio exterior de lácteos de ambos países DD ha tenido un contexto común en el MERCOSUR a partir de 1995, cuando el propio bloque, y en particular Brasil, absorbió hasta 1999 la mayor parte de los excedentes exportables de Argentina y Uruguay. Esta situación cambia en 1999 con la caída de las importaciones brasileñas y los dos países salen al contexto internacional a colocar sus productos, con las limitaciones que ello implica: distorsiones provenientes de políticas gubernamentales, restricciones para el acceso a muchos mercados y otros.

No obstante, al medir el desempeño competitivo con el índice de Balassa (IB), observamos tendencias crecientes en la competitividad para ambos productos (LPE y LPD) en los dos países para todo el periodo (gráficas 3 y 4). Sin embargo, para la LPD en Argentina se observa un leve crecimiento hasta el año 1999, comenzando a decrecer desde entonces, posiblemente como consecuencia de la situación de la caída de la demanda brasileña. El mayor crecimiento de la competitividad de LPE en Argentina se observa en toda la década de los noventa, cuando hubo fuertes inversiones locales en remodelaciones y construcción de nuevas plantas, tanto de empresas nacionales como extranjeras. Se observa una fuerte disminución a principios del siglo, cuando ocurre en 2001 la crisis nacional por la caída del plan de convertibilidad, y una fuerte devaluación que conduce después a una recuperación en 2004. Sin embargo, para la LPD la tendencia no es tan clara. Muestra un desempeño competitivo adverso en 1992 y una recuperación a partir de 1993, manteniéndose relativamente estable hasta 2003. Entonces empieza a decaer, con valores menores que la unidad en 2004 y, por tanto, llegando a revelar desventajas competitivas. Una situación de mayor interés en la exportación de LPE, tanto por condiciones del mercado internacional como por la eficiencia de la mejorada infraestructura de la industria local, podría motivar esta desventaja de la LPD. 
Gráfica 3

Evolución del desempeño competitivo revelado de Argentina en el comercio de leche en polvo. Indicador IB. Periodo 1990-2005

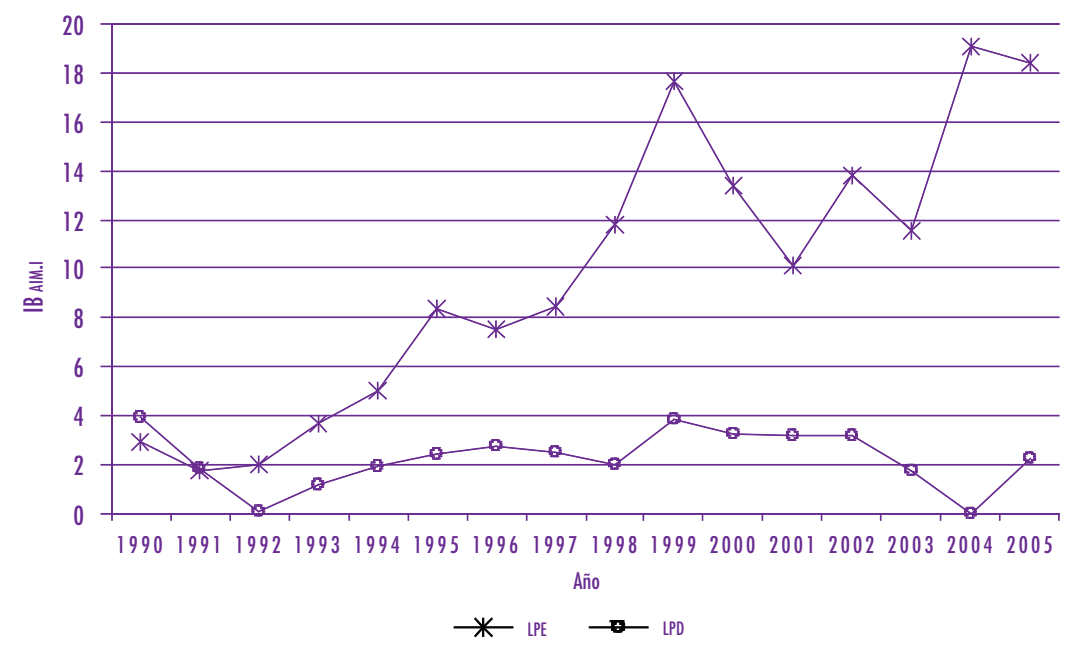

Fuente: Elaboración propia con base en datos de FAOSTAT y UNCTAD.

Para Uruguay, mientras que en la década de los noventa se observan valores mayores para la LPD que para la LPE, a partir de 1999 se revierte tal patrón, manifestándose una fuerte caída del IB para el primer producto y un gran aumento para el segundo. A partir de 2003, para ambos productos se revela una gran disminución en el indicador. Este resultado es llamativo, dado que después de la crisis uruguaya de 2002 y la devaluación las exportaciones de LPE se incrementaron notablemente desde 2004, aunque las de LPD sólo revierten la tendencia decreciente un año después.

El comportamiento del IB requiere profundizar más sobre las características del desempeño competitivo revelado por el indicador en lo que hace a los valores y a su evolución año tras año. Para ello podemos desagregar el IB en dos componentes: una "sectorial" y otra "global". Así se mide, por un lado, la competitividad internacional en términos de la participación mundial de las exportaciones de un determinado sector (en este caso la leche en polvo) y, por el otro, la participación mundial de las exportaciones totales, donde la combinación de ambas explicaría la especialización del país en el sector (De Benedectis et al., 2002). Esta posibilidad de separar la componente sectorial de la global es una propiedad específica del IB, resultante de desagregar su cómputo. Específicamente de (1), si se toma $\left(x_{i, l} / x_{M, l}\right)$, se está considerando la componente "sectorial", o sea la participación de las exportaciones de leche en polvo del país $i$ respecto a las exportaciones mundiales de leche en polvo; y por otra parte, el factor multiplicativo $\left(X_{M} / X_{i}\right)$ representa la inversa de la participación 
Gráfica 4

Evolución del desempeño competitivo revelado de Uruguay en el comercio de leche en polvo. Indicador IB. Periodo 1990-2005

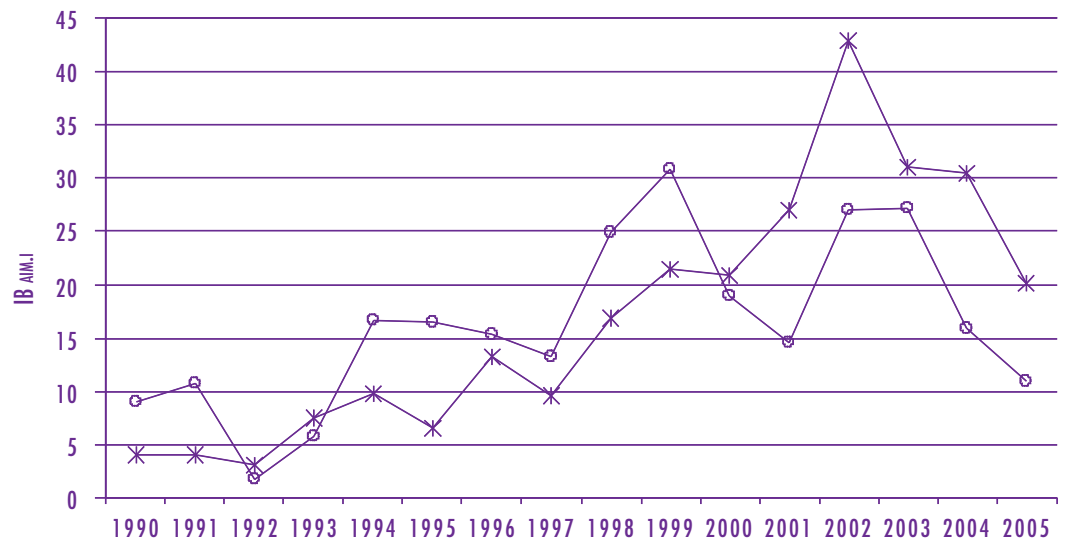

Año

$$
\rightarrow \text { LPE } \rightarrow \text { LPD }
$$

Fuente: Elaboración propia con base en datos de FAOSTAT y UNCTAD.

de las exportaciones totales realizadas por el país en relación con las exportaciones mundiales totales. El cuadro 1 muestra estas dos componentes para cada año en que comienza cada quinquenio.

Cuadro 1

Componentes "Sectorial" y "Global" de la competitividad

Comparaciones quinquenales

\begin{tabular}{|c|c|c|c|c|c|}
\hline & \multirow{2}{*}{$\begin{array}{c}\text { Componente } \\
i=\end{array}$} & \multicolumn{2}{|c|}{ LPE } & \multicolumn{2}{|c|}{$\angle P D$} \\
\hline & & Argentina & Uruguay & Argentina & Uruguay \\
\hline \multirow[t]{2}{*}{1990} & $\left(x_{i, l} / x_{M, l}\right)$ & 0.0103 & 0.0019 & 0.14 & 0.0044 \\
\hline & $\left(\dot{X}_{M} / \dot{X}_{i}^{\prime}\right)$ & 282.82 & 2063.66 & 282.82 & 2063.66 \\
\hline \multirow[t]{2}{*}{1995} & $\left(x_{i, l} / x_{M, l}\right)$ & 0.0341 & 0.0026 & 0.0099 & 0.0067 \\
\hline & $\left(X_{N} / X^{\prime}\right)$ & 246.57 & 2454.37 & 246.57 & 2454.37 \\
\hline \multirow[t]{2}{*}{2000} & $\left(x_{i, 1} / x_{M, l}\right)$ & 0.0548 & 0.0074 & 0.0135 & 0.0067 \\
\hline & & 244.32 & 2804.6 & 244.32 & 2804.6 \\
\hline \multirow[t]{2}{*}{2005} & $\left(x_{i, l} / x_{M, l}\right)$ & 0.0715 & 0.0066 & 0.0089 & 0.0036 \\
\hline & & 257.13 & 3029.15 & 257.13 & 3029.15 \\
\hline
\end{tabular}

Fuente: Elaboración propia con base en datos de flujos de comercio de FAOSTAT y UNCTAD. 
Al comparar la LPE y la LPD para cada país se observa que las participaciones de la LPE en las exportaciones mundiales son mayores que la participación de la LPD. Sin embargo, en Uruguay ocurre lo contrario en la década de los noventa, donde la participación de la exportaciones de LPD de Uruguay respecto a las mundiales es mayor que la participación de las exportaciones de LPE, revirtiéndose este orden para el periodo 2000-2005 y, por tanto, cambiando la componente sectorial hacia una mayor competitividad relativa en el sector de la LPE.

A su vez, se puede observar que la participación mundial de las exportaciones de leche en polvo (tanto LPE como LPD) de Argentina es en todos los casos mayores que la representada por Uruguay.

En términos de la componente global podemos observar que en Argentina la participación del valor de las exportaciones totales respecto al total exportado mundial es significativamente mayor que en el caso de las exportaciones uruguayas, lo que aumenta el valor del indicador IB para Uruguay. Asume un valor promedio aproximado de 16 contra 9.71 en LPE y 2.27 en LPD para Argentina.

Por tanto, al considerar el índice IB como medida de competitividad, sobre todo en términos de comparaciones de grado una vez que la demarcación ventajas/desventajas competitivas ha sido establecida, resulta de gran importancia tener presente la composición del indicador a fin de dilucidar la fuente de la diferencias entre el valor revelado por dos países competidores. Así en este caso pudimos ver que la componente sectorial de Argentina es mayor que la de Uruguay, lo que implica mayores valores del IB para el primero; sin embargo, al observar la componente global, esto es, la participación del país en el comercio global, la de Uruguay es menor, y al ser el inverso de esta participación una componente multiplicativa en el cómputo del IB, menores valores de la componente global implican mayores valores del IB. Así el valor del indicador de Uruguay se acrecienta con este factor.

En resumen, si se considera sólo al sector lechero, independientemente de los otros sectores productivos-exportadores del país, Argentina resulta más competitivo que Uruguay en el comercio de leche en polvo. Sin embargo, al tomar en cuenta la importancia relativa del sector dentro de la balanza global de cada país (i.e., la combinación de la componente sectorial y la global) Uruguay resulta más competitivo que Argentina en el sector, si se toma al indicador como una medida cardinal.

El cuadro 2 muestra una comparación entre ambas naciones mediante el cociente de los IB registrados para cada producto. A diferencia de la denominada componente "sectorial" que indica la participación del sector en un país respecto al mismo sector en el mundo, este cociente indica la participación del sector en una nación respecto a 
la otra. Por ejemplo, en 1995, la participación de las exportaciones de LPE argentinas fueron 1.3 veces mayores que las de LPE de Uruguay; sin embargo, para el 2000 tal participación sólo fue 0.64 de las uruguayas. Este patrón, en el que la importancia relativa del sector de leche en polvo es mayor en Uruguay que en Argentina, se repite en 1990 y en 2005, y en los cuatro años para el caso de LPD.

Cuadro 2

Comparaciones de la competitividad de Argentina y Uruguay por producto

\begin{tabular}{|ccc|}
\hline$I B_{A, l} / I B_{U / l}$ & & \\
$A \tilde{n} o l=$ & $L P E$ & $L P E$ \\
\hline 1990 & 0.72686 & 0.43863 \\
1995 & 1.30676 & 0.14972 \\
2000 & 0.64426 & 0.17453 \\
2005 & 0.91654 & 0.21147 \\
\hline
\end{tabular}

Fuente: Elaboración propia con base en datos de flujos de comercio de FAOSTAT y UNCTAD.

Por último, en el estudio de la evolución del desempeño competitivo de estos dos productos resulta de interés determinar la importancia relativa de uno en relación al otro. El cuadro 3 muestra el cociente de los IB de LPE y LPD para cada uno de los países tomando nuevamente sólo cuatro años del periodo considerado, separados en forma quinquenal. Tales cocientes indican la diferencia cuantitativa de la participación mundial de un país en el comercio de LPE respecto de la de LPD.

Cuadro 3

Comparaciones entre productos: LPE vs LPD

\begin{tabular}{|ccc|}
\hline$I B_{i, L P E} / I B_{i / L P D}$ & & \\
$A \tilde{n} \mathrm{i} i=$ & Argentina & Uruguay \\
\hline 1990 & 0.73401 & 0.44294 \\
1995 & 3.40687 & 0.39034 \\
2000 & 4.05916 & 1.09965 \\
2005 & 7.98749 & 1.84293 \\
\hline
\end{tabular}

Fuente: Elaboración propia con base en datos de FAOSTAT y UNCTAD.

Para Argentina puede observarse que mientras en 1990 la participación mundial de la LPE representa aproximadamente $70 \%$ de la participación mundial de la LPD, en 1995 esta situación cambia, manifestándose en una participación mundial de LPE 3.4 veces mayor que la de LPD. Aumenta en los años posteriores y, por tanto, cambia el perfil competitivo del sector hacia la LPE. Para Uruguay se observa una tendencia 
similar, algo menor, así como la diferencia entre ambos productos. Específicamente, para 1990 y 1995 la participación mundial de Uruguay en el comercio de LPD es mayor que la de LPE, mientras que en 2000 son aproximadamente iguales (valor del cociente cercano a la unidad), revirtiéndose para 2005 en el que la participación mundial de LPE es 1.84 veces más grande que la de LPD.

Con este análisis hemos podido comprobar que el fenómeno de la competitividad, medido con algún indicador específico, puede ser utilizado para realizar distintas comparaciones. Entre ellas, la desagregación del indicador entre su componente "sectorial" y "global" para cada producto y país en un año determinado; comparaciones entre ambos países para cada uno de los productos considerados, y comparaciones entre productos para cada nación. La conjunción de todos estos resultados nos permiten obtener una mirada más amplia de la evolución del desempeño competitivo.

En general, hemos observado con el IB tendencias crecientes con ciertas oscilaciones, mayores componentes sectoriales y globales en Argentina en relación con Uruguay, y mayores para la LPE que en la LPD para cada país. La conjunción de estas dos componentes permite concluir que la componente global gana en la determinación final de los valores del IB generando valores mayores $-\mathrm{y}$ por tanto un mayor grado de competitividad si la cardinalidad es interpretada en este sentido- en Uruguay que en Argentina. Esto se reafirma de las comparaciones entre países en donde en general la participación de las exportaciones de leche en polvo uruguayas respecto a las argentinas resultan mayores. Por último, al comparar ambos productos para cada nación, se observa una importancia relativa mayor de la LPE en Argentina a partir de 1995, mientras que en Uruguay los primeros años del periodo analizado se caracterizan por participaciones mundiales de la LPD mayor que la de la LPE, cambiando la situación a finales del periodo y, por tanto, concluyendo para ambos países un mejor desempeño competitivo en la LPE.

Hasta ahora, al utilizar el índice de Balassa, se consideró sólo un flujo comercial, específicamente el valor de las exportaciones. Sin embargo, si tomamos en cuenta las exportaciones netas, incluyendo el otro flujo del comercio, cabe preguntarse si las conclusiones realizadas siguen siendo válidas. En la sección anterior al presentar las diferentes metodologías para medir las ventajas competitivas se consideraron tres indicadores alternativos de exportaciones netas: el IVRC, el IMM y el IL. El cuadro 4 muestra un resumen sobre los valores de estos tres indicadores para cada producto y cada país.

Los montos de las importaciones de leche en polvo para ambos países son muy pequeños, cuando existen. Al considerarlas, los dos países en el periodo son competitivos, dado que en todos los casos el valor promedio para cada uno de los tres 
Cuadro 4

Resumen de los indicadores basados en las exportaciones netas Periodo 1990-2005

\begin{tabular}{lccccccc}
\hline & & \multicolumn{3}{c}{ Promedio 1990-2005 } & \multicolumn{3}{c}{ Coeficiente de variación (\%) } \\
& & IVCR & \multicolumn{1}{c}{ IMM } & IL & IVCR & IMM & $I L$ \\
\hline Argentina & & & & & & & \\
& LPE & 8.6402 & 0.6884 & 0.2464 & 79.4773 & 66.6909 & 72.0686 \\
& LPD & 1.8813 & 0.6556 & 0.0423 & 86.6898 & 81.0609 & 123.5435 \\
\multirow{5}{*}{ Uruguay } & & & & & & & \\
& LPE & 4.7505 & 0.9963 & 0.4899 & 35.219 & 0.6024 & 61.2810 \\
& LPD & 14.8247 & 0.9974 & 0.4054 & 50.1388 & 0.3243 & 38.4456 \\
\hline
\end{tabular}

Fuente: Elaboración propia con base en datos de flujos de comercio de FAOSTAT y UNCTAD.

indicadores es positivo. Para Argentina la variabilidad es mayor en todos los casos. Ello se debe en parte a que para esta nación existen años (1990-1992) para los cuales se revelan desventajas competitivas (valores negativos), tanto en el comercio de LPE como de LPD, mostrando a partir de 1993 una tendencia creciente en el caso de LPE y un comportamiento más errático para la LPD, aunque revelando ventajas competitivas en todos los demás años.

Sin embargo, en Uruguay se revelan ventajas competitivas en todos los años según los tres indicadores, y el comportamiento es relativamente estable en contraposición a la tendencia claramente creciente mostrada por el índice Balassa. Para este país el IMM muestra valores cercanos a la unidad con muy poca variación año tras año, lo que explica el bajo coeficiente de variación y un alto grado de competitividad, dado que alcanza el límite superior teórico de tal indicador. A su vez, el hecho de que la variabilidad del IL sea mayor a la del IVCR se debe a que el primero muestra una tendencia creciente más clara que el segundo y, por tanto, mayores valores del indicador, apaciguándose en los últimos años del periodo.

Por tanto, las conclusiones generales respecto al posicionamiento competitivo no cambian según lo revelado por los cuatro indicadores alternativos. Sin embargo, al observar aspectos de tendencias, las conclusiones difieren según se tome uno u otro indicador. Adicionalmente, se observan algunas diferencias entre el indicador basado en exportaciones y los basados en la balanza neta para el caso de Argentina en los primeros tres años de la década de los noventa, en el que el primero muestra ventajas competitivas mientras los otros revelan desventajas.

En este análisis de comparación de indicadores, implícitamente se consideran las conclusiones sobre las semejanzas o diferencias entre ellos con base en la interpreta- 
ción de las medidas en un sentido dicotómico, cuyos resultados son los de ventajas o desventajas. Adicionalmente, podemos compararlos en función del ordenamiento que el indicador establezca del desempeño competitivo revelado en cada año, como también como medida de grado de competitividad con base en el valor específico asumido por el índice. En la siguiente sección se presenta un análisis de consistencia de los indicadores a partir de estas tres interpretaciones de las ventajas competitivas reveladas.

\section{Consistencia de indicadores}

Según se ha explicado, se procede a hacer la prueba de consistencia de los indicadores. En primer lugar, en el cuadro 5 se presentan los resultados de la prueba de consistencia cardinal para ambos países y productos.

Cuadro 5

Consistencia cardinal. Coeficientes de correlación. Periodo 1990-2005

\begin{tabular}{|c|c|c|c|c|}
\hline & \multicolumn{2}{|c|}{ Argentina } & \multicolumn{2}{|c|}{ Uruguay } \\
\hline & $L P E$ & $L P D$ & $L P E$ & $L P D$ \\
\hline \multicolumn{5}{|l|}{ IB } \\
\hline IVCR & 0.9914 & 0.9255 & 0.5922 & 0.3301 \\
\hline IMM & 0.7709 & 0.7095 & 0.264 & -0.482 \\
\hline IL & 0.9463 & 0.8427 & 0.9901 & 0.8606 \\
\hline \multicolumn{5}{|l|}{ IVCR } \\
\hline IMM & 0.8435 & 0.9075 & -0.0842 & -0.0425 \\
\hline IL & 0.9695 & 0.9471 & 0.6117 & 0.099 \\
\hline \multicolumn{5}{|l|}{ IMM } \\
\hline II & 0.888 & 0.906 & 0.3124 & -0.2716 \\
\hline
\end{tabular}

Fuente: Elaboración propia con base en datos de FAOSTAT y UNCTAD.

Los resultados para Argentina muestran que el IB con el IVCR y el IL son altamente consistentes, mientras que el IB con el IMM lo son en menor medida (coeficiente menor a 0.80 pero mayor a 0.7$)$. Al considerar los indicadores basados en las exportaciones netas, todos resultan altamente consistentes entre sí destacándose el IVCR con el IL.

Las comparaciones por cardinalidad para el caso de Uruguay no son tan alentadoras. Entre el IB y el IVCR, ambos basados en el enfoque de ventajas comparativas reveladas, se observa muy poca consistencia, principalmente para la LPD, en donde el coeficiente de correlación es de 0.33 . Para este producto aparecen inconsistentes como medidas de grado el IB con el IMM y muy poco consistencia para la LPE. Sin embargo entre el IB y el IL, ambos indicadores construidos con base en diferentes 
enfoques teóricos, hay gran consistencia como medidas cardinales. Entre los indicadores netos se resalta el hecho de que el IMM es inconsistente tanto con el IB como con el IL. Ello se debe sobre todo a que para Uruguay este indicador muestra valores cercanos al límite superior, con escasa y, en algunos casos, nula variabilidad. Por tanto, como medida de grado, el IMM resulta poco conveniente para este país en particular. A su vez, entre el IVCR y el IL los resultados también muestran muy poca consistencia, principalmente para el caso de la LPD.

La prueba de consistencia ordinal (cuadro 6) indica que para Argentina los coeficientes para la LPE son mayores que los correspondientes a la prueba cardinal, o sea, se encuentra una mayor consistencia en términos ordinales. Para el caso de la LPD, los coeficientes son mayores a excepción de las pruebas entre el IVCR y el IMM (cuyo valor es de 0.82) y entre el IMM y el IL cuyo coeficiente indica una baja consistencia en términos ordinales (coeficiente igual 0.66).

Cuadro 6

Consistencia ordinal. Correlación por rangos. Periodo 1990-2005

\begin{tabular}{|c|c|c|c|c|}
\hline & \multicolumn{2}{|c|}{ Argentina } & \multicolumn{2}{|c|}{ Uruguay } \\
\hline & $L P E$ & $L P D$ & $L P E$ & $L P D$ \\
\hline \multicolumn{5}{|l|}{ IB } \\
\hline IVRC & 0.9941 & 0.9735 & 0.441 & 0.6588 \\
\hline IMM & 0.8824 & 0.7941 & 0.1552 & -0.521 \\
\hline IL & 0.95 & 0.9118 & 0.9882 & 0.8235 \\
\hline \multicolumn{5}{|l|}{ IVRC } \\
\hline IMM & 0.8941 & 0.8294 & 0.2956 & -0.1854 \\
\hline IL & 0.9588 & 0.9265 & 0.4029 & 0.3559 \\
\hline \multicolumn{5}{|l|}{ IMM } \\
\hline IL & 0.9812 & 0.6676 & 0.54 & -0.1766 \\
\hline
\end{tabular}

Fuente: Elaboración propia con base en datos de FAOSTAT y UNCTAD.

Al igual que la prueba ordinal, la cardinal para Uruguay indica consistencia sólo entre el índice de Balassa y el índice de Lafay. En este caso, se presentan inconsistencias para el caso de la LPD entre el IB y el IMM, el IB y el IMM, y entre el IL y el IMM, mostrando nuevamente la inconsistencia entre el índice de Michaely modificado y los demás indicadores.

En el cuadro 7 se presentan los resultados de la prueba de consistencia dicotómica, que reafirma las conclusiones obtenidas con la simple observación de los valores de cada año. 
Cuadro 7

Consistencia dicotómica. Proporción de años con igual demarcación Periodo 1990-2005

\begin{tabular}{lccccc}
\hline & & Argentina & & & Uruguay \\
& & LPE & LPD & LPE & LPD \\
\hline IB & & & & & \\
& IVRC & 0.8125 & 0.8765 & 1 & 1 \\
& IMM & 0.8125 & 0.8765 & 1 & 1 \\
& II & 0.8125 & 0.8765 & 1 & 1 \\
IVRC & & & & 1 \\
& IIM & 1 & 1 & 1 & 1 \\
& II & 1 & 1 & 1 & 1 \\
IMM & & 1 & 1 & 1 & 1 \\
\hline
\end{tabular}

Fuente: Elaboración propia con base en datos de FAOSTAT y UNCTAD.

Para Uruguay, a pesar de que para cierto par de indicadores aparecían inconsistencias en términos cardinales y ordinales, en términos dicotómicos todos los indicadores son perfectamente consistentes, es decir, todos coinciden en todos los años respecto al posicionamiento competitivo. Este resultado puede parecer trivial dado que Uruguay se caracteriza por tener un desempeño competitivo favorable en todos los años del periodo; sin embargo, existen divergencias entre las diferentes metodologías si el investigador las toma como medida de grado y orden.

En Argentina se observa consistencia perfecta entre los tres indicadores basados en las exportaciones netas, no así entre éstos y el Balassa a pesar de que pueden considerarse consistentes al tener coeficientes mayores a 0.8. Éstos se deben, como apuntamos antes, a que a principios de la década de los noventa el indicador de Balassa revela ventajas competitivas, mientras que al considerar el valor de las importaciones en el cálculo del indicador se revelan desventajas.

\section{Conclusiones}

La evolución del comercio internacional de leche en polvo de Argentina y Uruguay desde la decisión de integrar un bloque de libre comercio ha tenido altibajos, que no son coincidentes entre ambos países. Para evaluar la competitividad de cada uno en el periodo 1990-2005 se ha recurrido a varios indicadores alternativos: el índice de Balassa, el índice de ventajas relativas del comercio, el índice de Michely modificado y el índice de Lafay.

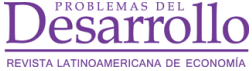


Desde el punto de vista metodológico se han encontrado diferencias en los resultados según el tipo de indicador utilizado. Al realizarse las pruebas de consistencia puede observarse que al tener en cuenta a los indicadores de ventajas competitivas como medidas cardinales y ordinales, la utilización alternativa de alguno de ellos genera complicaciones. En algunos casos, el resultado revelado, en cuanto al grado de competitividad y establecimiento de rango en aquellos años donde se consideran las mayores ventajas, difieren según se utilice uno u otro índice. Ello se presenta particularmente para el caso de Uruguay, donde si bien se revela un desempeño competitivo favorable en todo el periodo con una consistencia dicotómica perfecta entre los cuatro indicadores, sólo el IB y el IL son relativamente consistentes en términos ordinales como cardinales. Aparecen inconsistencias con el IMM y muy poca correlación para el IVCR, lo que lleva a recomendar cautela en la elección a quienes deseen utilizar esta metodología en el futuro. Si bien el indicador de Balassa supone la omisión de un flujo de comercio, lo que implica a priori una pérdida de información, su simple construcción permite extensiones en el análisis más allá del simple cómputo de una medida estimada de ventajas comparativas, como puede ser la descomposición en factores sectoriales y globales de la competitividad. Ello posibilita una aclaración sobre la fuente de determinación, en relación con cada uno de tales factores. A su vez, para el análisis comparativo entre productos y países, el IB permite extraer conclusiones claras y de fácil interpretación. Para este estudio en particular, la metodología de Balassa ha resultado de gran utilidad a fin de esclarecer los componentes de la competitividad. A pesar de que el índice IVCR es una extensión de aquél al comercio neto, éste supone una mayor inestabilidad de la medida, contribuyendo sólo en la inclusión de un flujo adicional sin mejorar (e incluso complicar) las bondades metodológicas de su primitivo. Por tanto, si es deseable obtener una medida que involucre tanto el lado de la demanda como de la oferta, resultan de mayor interés el IMM o el IL como medidas complementarias al IB. Ellos muestran un aspecto clave en la especialización comercial como lo es la evolución de la balanza comercial del sector lácteo en relación con la balanza comercial total. Dado que el índice de Lafay muestra una gran consistencia - en los tres sentidos - con el índice de Balassa, cosa que no ocurre con el índice de Michaely modificado, concluimos que los mejores indicadores para este caso de estudio son el IB y el IL como medidas complementarias. Es decir, como medidas que cuantifican el desempeño competitivo de manera similar y cuya inclusión permite la ampliación y el enriquecimiento de las conclusiones sobre la evolución de la competitividad en el comercio de leche en polvo de los dos países considerados.

Desde el punto de vista del desempeño competitivo, para la LPE se ha encontrado 
que Argentina se muestra competitiva durante los noventa, pero con una fuerte caída en 1999, y luego una recuperación desde 2003. Uruguay tiene un comportamiento bastante similar hasta ese año, pero, en lugar de decaer, presenta un fuerte incremento del indicador hasta 2003, en que recién comienza a disminuir.

En LPD Argentina no tiene una clara tendencia, alternando una mayoría de años con ventajas, pero aparecen otros con desventajas competitivas. Uruguay, que era fuertemente competitivo en este producto, lo sostiene hasta 1999, cuando se produce una inversión en su comportamiento. Desde aquí y hasta 2003 resulta más competitivo en LPE que en la LPD. A partir de este año ambos indicadores comienzan a declinar.

La caída en la competitividad de la lechería argentina y uruguaya en 1999 tiene como explicación más importante el desajuste producido por la disminución de la demanda brasileña como consecuencia de la devaluación producida en ese país, dada la dependencia que tenían de ese mercado. Brasil se había convertido en el principal socio comercial en estos productos, causando un fuerte shock en la lechería de los dos países, que debieron salir a buscar nuevos compradores en un mercado internacional bastante complicado. Con la caída del modelo de la convertibilidad en Argentina en 2001 se produce otra crisis que recién comienza a revertirse en 2003. Uruguay experimenta una recesión económica importante entre 1999 y 2002, con fuerte caída de rentabilidad agropecuaria hasta que se produce también una devaluación de su moneda.

En términos de implicación para las políticas públicas, los indicadores permiten relacionar cambios de las mismas con la competitividad del comercio exterior de cada país y, en este caso, en el contexto del MERCOSUR.

Así es como en primera instancia asociamos la constitución del MERCOSUR, su programa de liberalización comercial intrabloque para lácteos y la adopción de un arancel externo común desde 1995, con un mayor intercambio y una mejora en los indicadores de desempeño en LPE y LPD para ambos países analizados. Otra decisión política, que fue la devaluación brasileña sin que se alteraran los sistemas cambiarios de Argentina y Uruguay, también se asocia a la disminución en el valor de los indicadores. En tercer lugar, la crisis argentina y sus medidas devaluatorias, así como las de Uruguay en 2002, se visualizan con sus respectivos efectos sobre la competitividad sectorial.

Por último, vale la pena insistir en la importancia de la definición de políticas sectoriales que puedan mantenerse en el largo plazo, de manera que sus participantes tengan reglas claras para la toma de decisiones. Ésta es una condición crítica si realmente se quiere favorecer el desempeño competitivo en los países analizados. 


\section{Bibliografía}

Balance, Robert, Helmut Forstner y Tracy Murray, "Consistency test of alternative measures of comparative advantage", en The Review of Economics and Statistics, vol. 69, núm. 1, 1987, pp. 157-161.

Balassa, Bela, "Trade liberalization and revealed comparative advantage", en The Manchester School of Economic and Social Studies, vol 32, núm. 2, 1965, pp. 99-123.

De Benedictis, Luca y Massimo Tamberi, “A note of the Balassa Index of revealed comparative advantage", Working Paper, núm. 158, Macerata, Dipartimento di Economia, Universita' Politecnica delle Marche, 2002, pp. 1-37.

Depetris Guiguet, Edith, Rodrigo García Arancibia y Gustavo Rossini, "Ventajas comparativas reveladas en el comercio argentino de quesos: un análisis de sensibilidad y consistencia ante cambios en las referencias", en Anales de la $8^{\circ}$ Jornada de Investigación Desarrollo Institucional y Regional, Santa Fe, FCE, Universidad Nacional del Litoral, Soporte Digital, noviembre de 2008a.

, "Competitividad y especialización en lácteos. Un análisis de consistencia de indicadores", en Anales de la Asociación Argentina de Economía Agraria, Montevideo, soporte digital, noviembre de 2008b.

y Osvaldo Cappellini, El MERCOSUR lácteo: Evolución del proceso de integración, Santa Fe, JIPL, 1998, pp. 1-290.

Fertö, Imre y Lionel Hubbard, "Competitiveness and comparative advantage in Hungarian agriculture" in Mühelytanulmányok, Budapest, Discussion Papers, New Series 2001/2, 2001, pp. 1-12.

,"Revealed comparative advantage and competitiveness in Hungarian agri-Food sectors", in Technology Foresight in Hun- gary, Budapest, Institute of Economics, Hungarian Academy of Sciencies, 2002, pp. 1-16.

Gual, Jordi y Carmela Martin, "Trade and foreign direct investment with Central and Eastern Europe: Its impacts on Spain", en Faini R.y Portes R. (eds.), EU Trade with Eastern Europe: Adjustment and Opportunities, Londres, CEPR, 1995.

Lafay, Gerard, "The measurement of revealed comparative advantages", en M. G Dagenais and PA Muet (eds.), International Trade Modelling, Londres, Chapman \& Hall,1992, pp. 209-34.

Lagnevik, Magnus y Eamonn Pitts, "What determines food industry competitiveness?", en Traill B. \& Pitts E. (eds.), The Competitiveness in the Food Industry, Londres, Chapman \& Hall, 1997, pp. 1-34.

Laursen, Keld, "Revealed Comparative Advantage and the alternatives as measures of international specialization", DRUID Working Paper, 98-30, Copenhagen, DRUID, 1998, pp. 98-30.

Scott, Linda and Vollrath Thomas, "Global Competitive Advantages and Overall Bilateral Complementarity in Agriculture", Statistical Bulletin, núm. 850, Washington, DC, US Department of Agriculture, Economic Research Service, 1992, pp. 1-214.

Vollrath, Thomas, "A theoretical evaluation of alternative trade intensity measures of revealed comparative advantage", Weltwirtschaftliches Archiv, núm.127, vol. 2, 1991, pp. 265-280.

Zaghini, Andrea, "Trade advantage and especialization dynamics in acceding countries", Working Paper, núm. 249, Working Papers Series, Frankfurt, European Central Bank, 2003, pp. 1-52. 